\title{
Theoretical spectral scattering of Ice Clouds in the Near-Infrared
}

\author{
James E. Hansen and Howard Cheyney
}

\section{GPO PRICE \$ CFSTI PRICE(S)}
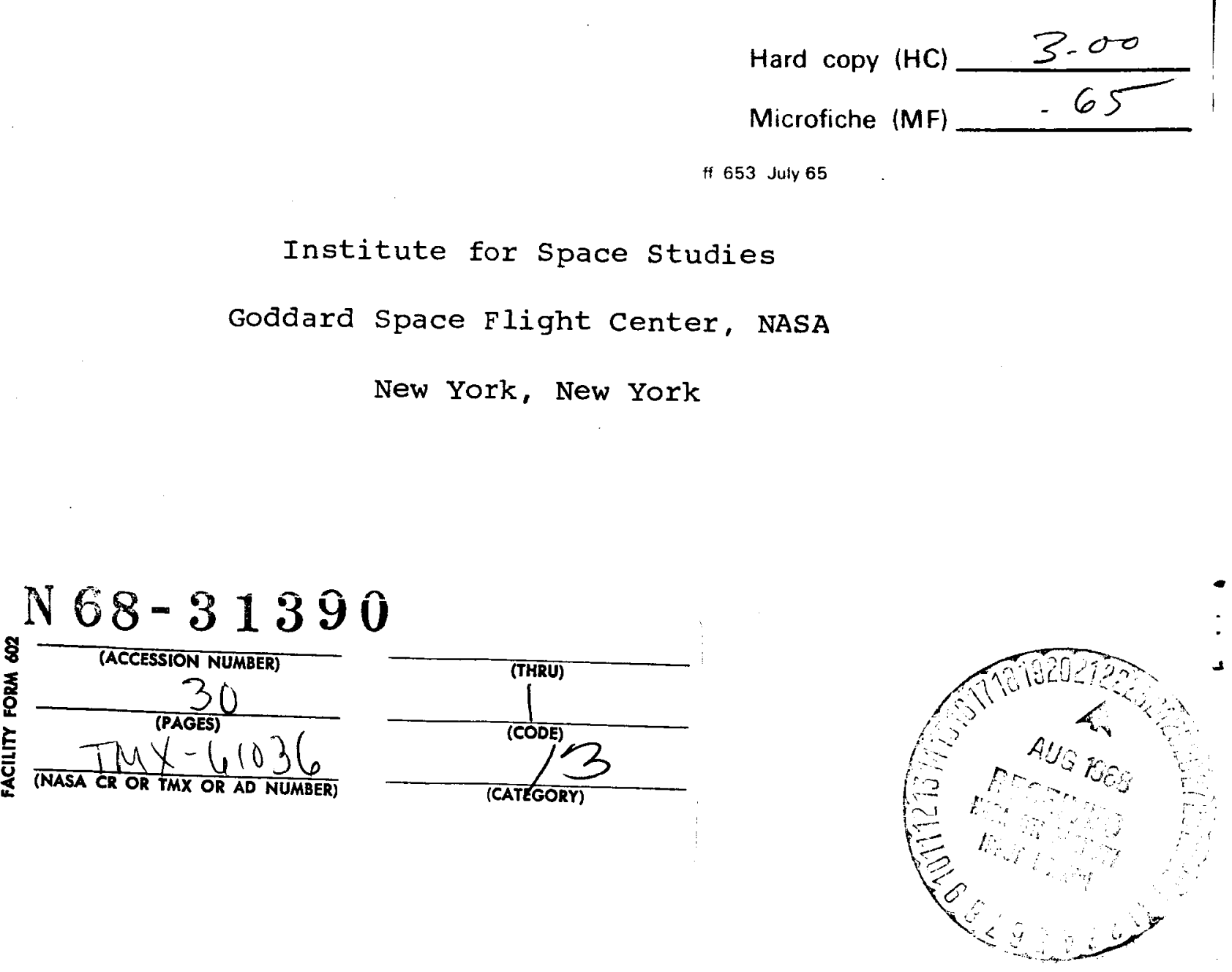


\section{ABSTRACT}

The spectral reflectivity of clouds of ice spheres is calculated for the near infrared wavelength region $(1 \mu \leqslant \lambda \leqslant 4 \mu)$ for comparison with laboratory observations of ice clouds. The laboratory cloud particles were highly nonspherical ice crystals, however, it is shown that the major feature of the theoretical spectrum are in close agreement with the observations when spheres of equal volume are employed in the radiative transfer problem. The results provide some support for the contention that randomly oriented nonspherical particles may be replaced by equivalent spheres for calculations of intensities.

The reflectivities are shown to be sensitive to the particle size, especially the reflectivity minima near 1.5 and $2.0 \mu$. Changes by a factor of two in the particle size cause significant variations in the major reflectivity features; this verifies that spectral reflectivity measurements can be of considerable value for cloud identification. The agreement of the theoretical and experimental spectra also indicatesthat it should be possible to obtain meaningful conclusions from infrared reflectivity measurements of planetary atmospheres, such as the observations which have been made on the atmosphere of Venus. 


\section{INTRODUCTION}

In the wavelength region from 1 to 4 microns the absorbtivity of ice, as well as that of many other materials, has an absolute value such that the reflectivity of clouds composed of micronsized particles should show large variations with wavelength and with particle size. Laboratory measurements confirm this and hence suggest that near-infrared reflectivities may be useful for the identification of clouds in planetary atmospheres; it is conceivable, for example, to derive the particle composition and size and the cloud optical thickness from the spectral reflectivity. Sagan and Pollack (1967), and later Hansen and Cheyney (1968), have attempted to extract such cloud parameters from a comparison of theoretical computations to atmospheric observations; however, to determine whether such a procedure can yield valid results it is important to find if the computations can in fact duplicate laboratory spectra in which the cloud parameters are known.

The primary purpose of this paper is to show that the major features in the best existing laboratory spectra can be duplicated by theoretical calculations. The results also indicate the sensitivity of the near-infrared reflectivities to variations of the cloud parameters.

Several authors have previously made computations for single scattering by cloud particles in the near-infrared. A two-stream approximation for the multiple scattering has been developed by 
Sagan and Pollack (1967) and applied to the near-infrared reflectivity of Venus. Good observational spectra in the nearinfrared have been published both for the atmosphere of venus (Bottema et al., 1965; Kuiper, 1962) and for terrestrial clouds (Blau et al., 1966).

\section{LABORATORY OBSERVATIONS}

Zander (1966a, b) made laboratory measurements which are well suited for a comparison to theoretical computations. The experimental methods he employed and a detailed description of his results will be published elsewhere [Zander, 1968]; hence in this section only the experimental conditions which most directly affect the theoretical interpretation will be given.

Zander measured the near-infrared spectral reflectivity of an optically dense ice cloud at an angle of incidence $\Delta_{0}=15^{\circ}$ from the normal, an angle of reflection $A=15^{\circ}$, and an azimuth angle difference $c_{0}-n_{0}=0^{\circ} ; \mathrm{i} . e .$, the measurements were for the specular direction. Evidence of a specular component in the diffuse reflection from terrestrial clouds and hazes has been reported (Tank, 1964; Deirmendjian, 1968) and hence observations in that direction may be an undesirable special case. However in the laboratory measurements it is unlikely that there was significant reflection from flat surfaces of horizontally 
oriented crystals because the particles were small and continually tumbling about [Zander, 1966b].

Zander (1968 and personal communication) sampled the particle shape and the size distribution by inserting a slide vertically into the upper part of the cloud and allowing the particles to adhere to the slide as a result of their own motion; this procedure could conceivably have led to a distribution biased toward the smaller particles as a result of their greater Brownian motion, but we have not made an analysis of that point. Zander found the individual particle diameters to be within the range $1 \mu \leq a \leq 3 \mu$ with the maximum of the distribution at $1.7 \mu$. About $1 / 3$ of these single crystals were prisms and most of the others were hexagonal plates. Each of these two forms showed cavities which resulted in a void space of not more than $\sim 20 \%$. Zander (1966a) reported that the particles which were clustered into aggregates composed a minimum fraction of those sampled, but, as will be shown below, the smaller number of aggregates were primarily responsible for the scattering properties of the cloud.

The absolute reflectivity was measured as $78 \pm 58$ at $\lambda=1.75 \mu$ and $56 \pm 58$ at $\lambda=2.25 \mu$ with the numbers being 
the ratio of the observed intensity to that of a Lambert surface with albedo unity. The observed relative reflectivity from $.95 \mu$ to $4.0 \mu$ is shown as the heavy curve in Figures 5, 6 and 8 . That curve represents the average of 5 runs; the individual runs did not depart from the average by more than $\sim 5-78$ with the largest variations being for $.95<\lambda<1.2 \mu$ and $3.0<\lambda<4.0 \mu$.

The optical thickness of the cloud in the visible region was measured at different times by zander and found to be in the range $\tau=24 \pm 3 . \tau(\lambda)$ is here defined such that $\exp (-\tau)$ is the fraction of vertically incident radiation which experiences neither absorption nor scattering while traversing the cloud.

The maximum in the observed reflectivity between 1.5 and $2.0 \mu$ appears to be at $\lambda \sim 1.8 \mu$ in zander's (1966b) graph but the corresponding minimum in the absorbtivity of ice is at $\lambda \sim 1.85 \mu$. It is not obvious that either the reflectivity measurement or the absortivity measurement should be in error by as much as $\Delta \lambda$ $\sim .05 \mu$, but the discrepancy probably results in part from Zander's finite resolving power $(\Delta \lambda \sim .04 \mu)$ and the fact that the reflectivity falls off more sharply from 1.85 to $2.0 \mu$ than from 1.85 to $1.5 \mu$. In order to have an uncluttered comparison of theory and observation we have drawn zander's curve with the relative maximum at $\lambda=1.85 \mu$. 
COMPUTATIONAL PROCEDURE

The Mie scattering computations for the phase function (scattering diagram) and the single particle albedo $\omega_{0}$ were made with standard methods for particle size distributions specified below. The optical constants (i.e., the complex index of refraction, $n_{r}-i n_{i}$ ) for ice were taken from the tables of Irvine and Pollack (1968) which are based on an extensive survey of the literature. It is expected that the error in $n_{i}$ may easily be $\sim 20 \%$. No temperature correction was applied to the tabulated quantities since the tables are for a temperature $\left(-50^{\circ} \mathrm{C}\right)$ sufficiently close to that in Zander's experiment $\left(-44^{\circ} \mathrm{C}\right)$. The phase function was calculated at 96 angles $\left[0(.2) 5(2.5) 180^{\circ}\right]$. The single particle albedo, $\omega_{0}$, was determined to a high accuracy $( \pm .00001)$ because for albedos near unity the variations in this parameter are magnified greatly by the multiple scattering.

The radiative transfer problem was solved using the "double only" method described by Hansen (1968). At each wavelength the phase function was used to determine the reflection and transmission functions for a cloud of optical thickness $\tau \sim 2^{-25}$ and the corresponding functions for a layer of twice that thickness were then obtained from the doubling equations, which are an expression of a principle first given by van de Hulst (1963). 
The process was repeated until an optical thickness $\tau \sim 2^{7}=128$ was reached.

The phase functions were expanded in cosines of the azimuth angle and the first 10 terms in the expansion were used; since the incident and emergent angles were near normal in Zander's experiment the omitted terms did not affect the first three figures of accuracy in the results. Hence the primary limitation on the accuracy was the number of polar angles used in the Gauss quadratures; this number was varied and chosen in each case to be such that the self consistency (and other checks) indicated the errors to be $<.1 \%$.

The computations were made at 25 wavelengths in the interval $.95 \mu \leq \lambda \leq 4 \mu$ including each wavelength in that region at which the absorbtivity has a relative maximum or minimum.

\section{COMPUTATIONAL RESULTS}

The initial computations were made for the particle size suggested in Zander's (1966a) original article, i.e., the particle diameters were taken to be in the range $1 \mu \leq d \leq 3 \mu$ with the peak of the distribution at $d_{m}=1.7 \mu$. The exact shape of the distribution was unknown but due to its narrowness this was unimportant; for the calculations it was chosen to follow the "cloud" model distribution of Deirmendjian (1964) 
$n(d) \propto d^{6} e-\frac{6 d}{d_{m}}$

where $\mathrm{n}(\mathrm{d})$ is the volume concentration at diameter $d$. This size spectrum is shown as distribution 1 in Figure 1 , the single scattering albedo and the relative cloud optical thickness as functions of wavelength are given in Figures 2 and 3 , and some representative phase functions are shown in the right half of Figure 4.

To permit an absolute comparison to Zander's observations the theoretical computations were made for

$$
\frac{S\left(\tau ; \theta, \phi ; \theta_{0}, \phi_{0}\right)}{4 \cos \theta \cos \theta_{0}}
$$

with $\theta=\theta_{0}=15^{\circ}$ and $\phi-\phi_{0}=0^{\circ}$, where $S$ is the scattering function in the definition of Chandrasekhar (1960). The theoretical reflectivities for three optical thicknesses of the cloud are compared to the observations in Figure 5. The observed absorption features near $\lambda=1.5,2.0$ and $3.0 \mu$ are present in the theoretical curves but the spectral reflectivity does not correspond closely to the observations: the major absorption features at 1.5 and $2.0 \mu$ are less than half as strong as observed by $\mathrm{Zander,} \mathrm{the} \mathrm{minor} \mathrm{features} \mathrm{near}$ 1.0 and $2.5 \mu$ are entirely absent, and the reflectivity is a factor of 3-4 too high in the $3.4-4.0 \mu$ range. The agreement would be somewhat improved if the observed curve were shifted in the vertical direction but a significant 
shift is not allowed within the absolute error estimated by Zander $( \pm 5 \%)$.

Perhaps the most likely cause of the discrepancy is the size assumed for the particles. Larger particles would cause stronger absorption features, and hence, to test this effect, calculations were made with particles a factor of two larger than those in the first distribution employed. The computed reflectivity was changed in the desired sense, but to a limited extent, and the fit to the observation was still unacceptably poor.

However, after the above computations were completed it was learned from Zander (personal communication) that the number of particles clustered into aggregates was significant ( $20 \%$ with an aggregate counted as a single particle). This is important since the components of an aggregate would not be expected to scatter incoherently and hence they should not be treated as individual particles in the radiative transfer calculations. The manner in which the aggregates should be approximated depends on the ratio of the particle size to the wavelength and on the particle morphology including the amount of void space. According to Zander (personal communication) the distribution of aggregates peaked at diameter $9 \mu$ with some particles being as large as $15 \mu$ and the aggregates were observed to be closely packed with a void space estimated as $<20 \%$. Since the particles were tightly packed it may be a reasonable 
approximation to treat them as spheres, even though they were not small compared to the wavelength. The diameter of the equivalent spheres would be slightly $(\sim 5 \%)$ less than the diameter of the aggregates but this fact would probably be at least balanced by the fact that $\mathrm{Z}$ ander found the particle diameter to increase with depth into the cloud. Therefore, calculations were made with a double cloud model size distribution (distribution 2, Figure 1) with $20 \%$ of the particles under the partial distribution peaking at diameter $9 \mu$. The single scattering albedo and the cloud optical thickness as a function of wavelength are shown in Figures 2 and 3. Some representative phase functions are shown in the left half of Figure 4 and the cloud reflectivity is given in Figure 6 .

The theoretical spectrum for the size distribution including the aggregates agrees quite well with the observational reflectivity and the required optical thickness is within the range specified by zander $(\tau=24 \pm 3)$. We note the following points:

The major features in the reflectivity from 1.3 to $2.2 \mu$ fit the observations almost exactly. These features are very sensitive to the particle size; computations with still larger particles [the 'cloud'model with its peak at diameter $16 \mu$ (distribution 3, Figure 1) for which representative phase functions are given in Figure 7j show features (Figure 8) significantly stronger than those for distribution 2. The absolute reflectivity with these larger 
particles is too small to be compatible with the observed curve.

The mean extinction diameter is defined by

$$
\bar{d}=2 \bar{r}=\frac{2 \int_{r_{1}}^{r_{2}} r(r) Q_{\text {ext }}(r, \lambda) d r}{\int_{r_{1}}^{r_{2}} n(r) Q_{\text {ext }}(r, \lambda) d r}
$$

where $Q_{\text {ext }}(r)$ is the extinction cross section for a particle of radius $r$, and $r_{1}$ and $r_{2}$ are respectively the lower and upper limits of the size distribution. $\bar{d}$ has the values $2.16,10.4$ and $20.8 \mu$ at $\lambda=.95 \mu$ for distributions 1,2 and 3, respectively. Hence easily measurable variations in the reflectivity may occur for changes of less than an order of magnitude in the characteristic particle size.

To further assess the relative contributions of the aggregate and individual particles, computations were made with a size distribution representing only the aggregates (a 'cloud' model with its peak at diameter $9 \mu, d_{1}=2 r_{1}$ $=5 \mu, \mathrm{d}_{2}=2 \mathrm{r}_{2}=15 \mu$ ). The results (not shown) were practically indistinguishable from those for distribution 2 (which had 208 aggregates). The reflectivities in these two cases differed by $<3 \frac{3}{2}$ at each of the 22 common wavelengths at which computations were made, with the reflec- 
tivity slightly lower for the distribution of aggregates only. The mean extinction diameter for the latter distribution was $11.0 \mu$ at $\lambda=.95 \mu$. These results suggest that the reflectivity features depend mainly on the large particles in the size distribution as a consequence of the fact that those particles largely determine the mean particle size.

The minor absorption feature at $1.03 \mu$ is hardy visible in any of the theoretical spectra and it is unlikely that employing nonspherical particles would significantly strengthen this depression. Hence it appears that the theoretical computations will not yield a feature as strong as observed by Zander unless the absorbtivity of ice has a greater relative maximum at $1.03 \mu$ than is indicated by the tabulated optical constants. $\lambda=1 \mu$ is in fact near the short wavelength limit of reliable determinations of the ice absorbtivity because of the high transmission there, however, the absorbtivity at $1.03 \mu$ would have to be approximately an order of magnitude larger than the value given by Irvine and Pollack (1968) in order to account for the feature reported by zander. Perhaps it is more likely that the observed reflectivity feature was an instrumental effect, since, according to W. Plummer (private communication), the wavelength was near the tail of emission of Zander's infrared source. Absorption by water vapor probably could not have been responsible for the feature because the water vapor absorption coefficient is much less at $1 \mu$ than 
at some longer wavelengths where no absorption was apparent. The minor feature at $2.6 \mu$ is considerably less prominent in the theoretical spectra than in the observed curve. Zander found this feature to vary significantly with temperature (probably due to changes in the particle size distribution) but there is clearly a discrepancy between the theory and the observations; this is apparently the result of an error in either the tabulated absorbtivity of ice or the assumptions employed in our computations.

In Figure 6 the theoretical reflectivity exceeds that observed in the range $3.6<\lambda<4.0 \mu$, but that is the region in which the observations are most uncertain. If the deviation is real, though, it could be a consequence of the spherical particle approximation since, as mentioned by D. G. Rea (personal communication), aggregates might scatter as large particles at long wavelengths and exhibit scattering properties characteristic of the smaller components on the aggregate surface at short wavelengths where $x=\frac{2 \pi r}{\lambda} \gg 1$. Such a scattering behavior has in fact been observed for mineral samples in the laboratory by Rea, but the possible discrepancy in the cloud reflectivities must be reconfirmed experimentally before it is taken as clear evidence for a breakdown in the spherical particle approximation.

The observed reflectivity of $3-4$ from 3.0 to $3.2 \mu$ is perhaps indicative of the noise level in Zander's experiment since any particles large enough to yield the 
observed features at 1.5 and $2.0 \mu$ would have a reflectivity $<18$ for $3.0 \leq \lambda \leq 3.2 \mu$

Our computed results shown in Figures 5, 6 and 8 may be compared to the theoretical computations of Sagan and Pollack (1967) who employed a two-stream approximation for the multiple scattering. When their reflectivity curves for single particles of given diameters are compared to our curves for size distributions with corresponding mean extinction diameters, it appears that the spectral features are somewhat more pronounced in our curves; this, however, is expected since their results apply to the intensity integrated over all angles of incidence and reflection and not to the intensity at the particular angles of incidence and emergence in Zander's experiment (which were nearly normal to the cloudtop). It is apparently for this reason that Sagan and Pollack obtain their closest agreement to the spectrum of Venus (which is practically identical to Zander's laboratory curve for particles of diameter $\sim 20 \mu$ while we get the best fit to Zander's laboratory data with particles of half that size. A preliminary comparison of our calculated spherical albedos to their results suggests that their approximation is quite accurate for the integrated intensity.

\section{DISCUSSION}

The computed results indicate the sensitivity of the near-infrared reflection spectrum to the particle size and 
to the cloud optical thickness; this reemphasizes the potential use of reflection spectra for cloud identification. Our computations were made at rather widely spaced wavelengths and no attempt was made to degrade the theoretical spectra to account for the finite resolving power $(\sim .04 \mu)$ in the observations; however, these deficiencies may easily be eliminated in cases where more exact computations are warranted.

Due to the uncertainties in the optical constants of ice and our incomplete knowledge of the experimental parameters such as the particle size distribution, it is possible that the close agreement of the major features in the theoretical and observational spectra is partly fortuitous. But until further observations and computations are made the results must be regarded as evidence that in some circumstances randomly oriented nonspherical particles may be approximated by spheres; this is a contention which has previously been made by Sagan and Pollack (1967) and others. However, since the scattering properties of the cloud were determined mainly by the aggregates which may have been more spherical than the individual plates and prisms, it would also be desirable to have reflectivity measurements from clouds in which the crystals were not allowed to agglomerate. From the minor reflectivity features there is a suggestion that the optical constants could be in error near $2.6 \mu$ and perhaps also near $1 \mu$; hence it would be of interest to have the absorbtivities remeasured in those regions. It 
would also be helpful to have the cloud reflection spectra reexamined at the same wavelengths.

Zander's measurements were the first of this type and at the time he performed them there were no theoretical calculations available; it is hence surprising that he obtained so many parameters which must be known for an adequate interpretation (such as the cloud optical thickness, the absolute reflectivity, the particle morphology and the size distribution). In future observations it will be important to control the experimental conditions as closely as possible, especially the particle size distribution which should be known at increments of the radius $\sim 1 \mu$.

On the whole the agreement between the laboratory and theoretical results is sufficiently close to encourage the comparison of similar computations to observations of atmospheric clouds. Measurements from an aircraft of the absolute intensity spectra of solar radiation scattered by terrestrial ice and water clouds have been reported by Espinola and Blau (1965) and $\mathrm{Blau}$ et al (1966) in the region $1.15-3.6 \mu$, including a few examples of the angular dependence of the scattered light. In their observations the absorption by $\mathrm{CO}_{2}$ and $\mathrm{H}_{2} \mathrm{O}$ vapor above and within the clouds is significant and must be accounted for in a theoretical interpretation; we will hence only mention here that the spectral features that they observed for cirrus clouds near $\lambda=1.5$ and $2.0 \mu$ are deep and appear to require particle sizes at least $10 \mu$ 
in diameter. Further observations including the angular pattern of the scattering, especially in the region $1<\lambda$ $<2.5 \mu$ where gaseous absorption is small, would be helpful for making a complete analysis.

Finally, the results of this paper also suggest that it is possible to obtain meaningful conclusions from the theoretical interpretations of the near infrared reflectivity of Venus (Sagan and Pollack, 1967; Hansen and Cheyney, 1968). 
ACKNOWLEDGMENTS

We would like to thank Dr. R. Jastrow, director of the Institute for Space Studies, for his hospitality. We are indebted to $\mathrm{Dr} . \mathrm{R}$. Zander for detailed correspondence on his experimental work and for furnishing a valuable preprint, to Dr. J. Pollack for helpful discussions and for originally suggesting the importance of comparing theoretical computations to Zander's observations, and to Dr. A. Arking for constructive comments on a draft version of this paper. We would also like to thank Drs. D. Deirmendjian, W. Irvine, W. Plummer, J. Potter, D. Rea and S. Ueno for useful suggestions. 


\section{REFERENCES}

Blau, H. H. Jr., R. P. Espinola, and E. C. Reifenstein, III, Near infrared scattering by sunlit terrestrial clouds, Appl. Opt. , $5,555,1966$.

Bottema, M., W. Plummer, J. Strong, and R. Zander, The composition of the Venus clouds and implications for model atmospheres, J. Geophys. Res., 70, $4401,1965$.

Chandrasekhar, S., Räıliative Transfer, p. 20, Dover, New York i960.

Deirmendjian, D., Scattering and polarization properties of water clouds and hazes in the visible and infrared,

Appl. Opt. , 3, 187,1964

Deirmendjian, D., Sun's image on airborne ice crystals?, Appl. Opt. , I, 556, 1968

Espinola, R. P., and H. H. Blau, Jr., Cloud composition from infrared spectra, J. Geophys. Res., 70, 6263,1965 .

Hansen, J. E., Radiative transfer by doubling very thin layers, to be published, 1968.

Hansen, J. E., and H. Cheyney, Near infrared reflectivity of Venus and ice clouds, J. Atmos. Sci., 25, 1968 (in press). 
Hulst, H. C. van de, A new look at multiple scattering, NASA Institute for Space Studies report (New York) 81 pp., 1963.

Irvine, W. M., and J. B. Pollack, Infrared optical properties of water and ice spheres, Icarus, 8, 324, 1968. Kuiper, G. P., Infrared spectra of stars and planets, 1: photometry of the infrared spectrum of Venus, 1-2.5 microns, Comm. Lunar and Planetary Lab., $1,83,1962$.

Tank, W. G., Optical phenomena in ice crystal clouds, AIAA paper, No. 64-312, $11 \mathrm{pp} ., 1964$.

Zander, R., Spectral scattering properties of ice clouds and hoarfrost, J. Geophys. Res., 71, 375, 1966a Zander, R., in Infrared balloon astronomy, AFCRL, 115 Pp., 1966b. [Def. Doc. Center, AD643-567,\$3.00.]

Zander, R., Additional details on the near infrared reflectivity of laboratory ice clouds, to be published, 1968. 


\section{F IGURES}

Fig. 1. Cloud particle size distributions normalized to unity at their maximum points. The single and multiple scattering results in Figs. 2-8 were made for these three size distributions of spherical ice particles. Distributions 1 and 2 coincide for diameters less than $3 \mu$. Fig. 2. Single scattering albedo $\left(\omega_{0}\right)$ for the three particle size distributions shown in Fig. 1. The wavelengths at which the single and multiple scattering computations were made are indicated by dots on the curve for distribution 3 .

Fig. 3. Cloud optical thickness normalized to unity at $\lambda=.95 \mu$ for the three particle size distributions shown in Fig. 1. The interpolated curves between the wavelengths of the computations (indicated on curve 3) may not be accurate for $2.8 \mu<\lambda<3.2 \mu$, but that is unimportant for the multiple scattering results (Figs. 5, 6, 8) since the intensity is so low in that wavelength region.

Fig. 4. Single scattering phase functions (scattering diagrams) normalized to unity, at representative wavelengths in the near-infrared for two of the particle size distributions shown in Fig. 1. The vertical scale for each successive wavelength is displaced by an order of magnitude; the wavelengths to which the numbers on the 
vertical scale apply are indicated in parenthesis.

Fig. 5. Near-infrared reflectivity of an ice cloud. The solid curve was observed in the laboratory with a resolution $\sim .04 \mu$ by zander. The theoretical curves are for the particle size spectrum without aggregates (distribution 1 , mean extinction diameter $\sim 2.16 \mu)$. The optical thickness applies to $\lambda=.95 \mu$. The reflectivity is defined as the ratio of the observed or theoretical intensity at the angles in Zander's experiment to the intensity from a perfect Lambert surface at the same angles.

Fig. 6. The same as Fig. 5 with the theoretical curves for the particle size spectrum including aggregates (distribution 2 , mean extinction diameter $\sim 10.4 \mu$ ). Fig. 7. Same as Fig. 4 for size distribution 3. Fig. 8. The same as Fig. 5 with the theoretical curves for the particle size spectrum of very large particles (distribution 3, mean extinction diameter $\sim 20.8 \mu$ ). For $\lambda>2.8 \mu$ the three theoretical curves are indistinguishable. 


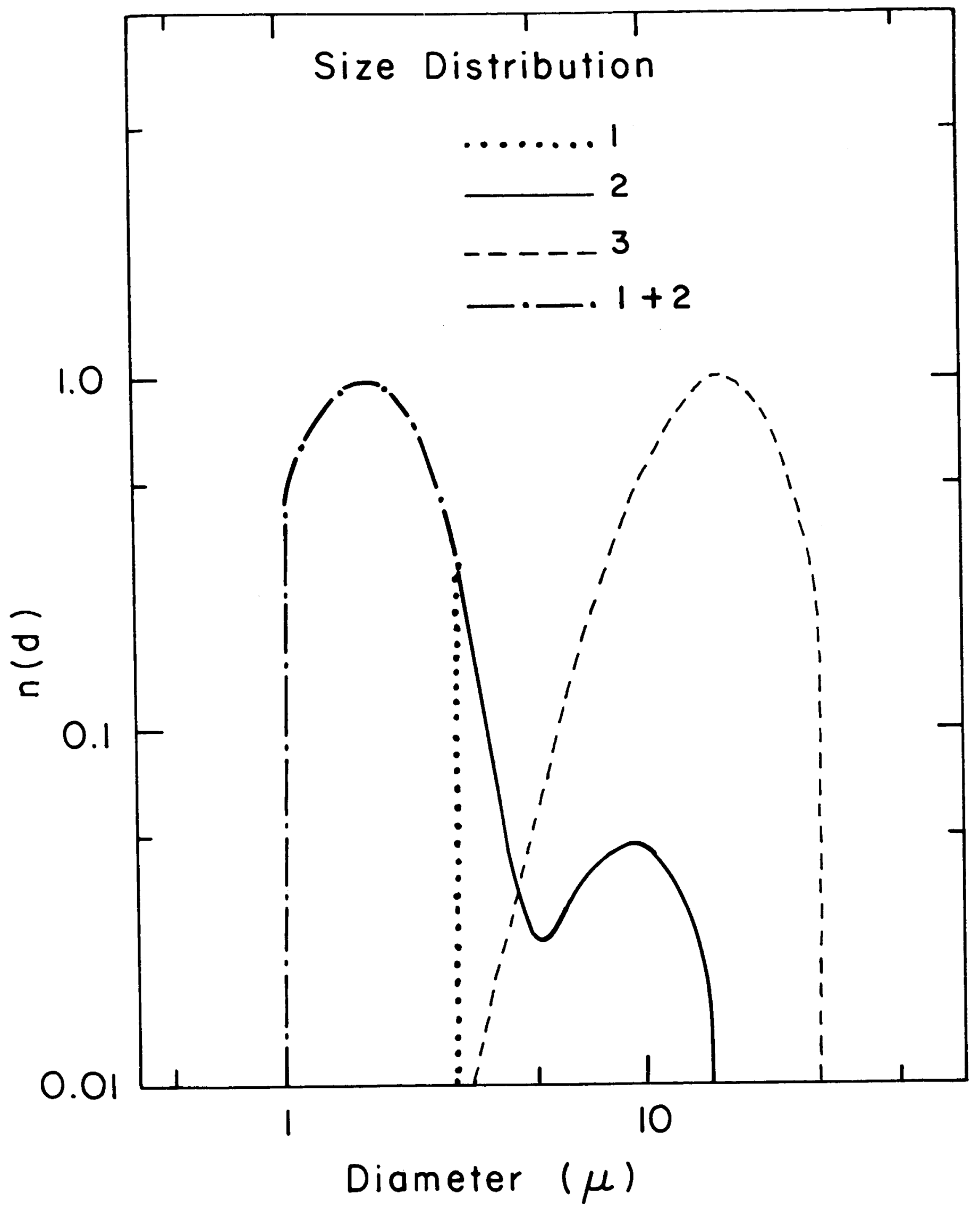




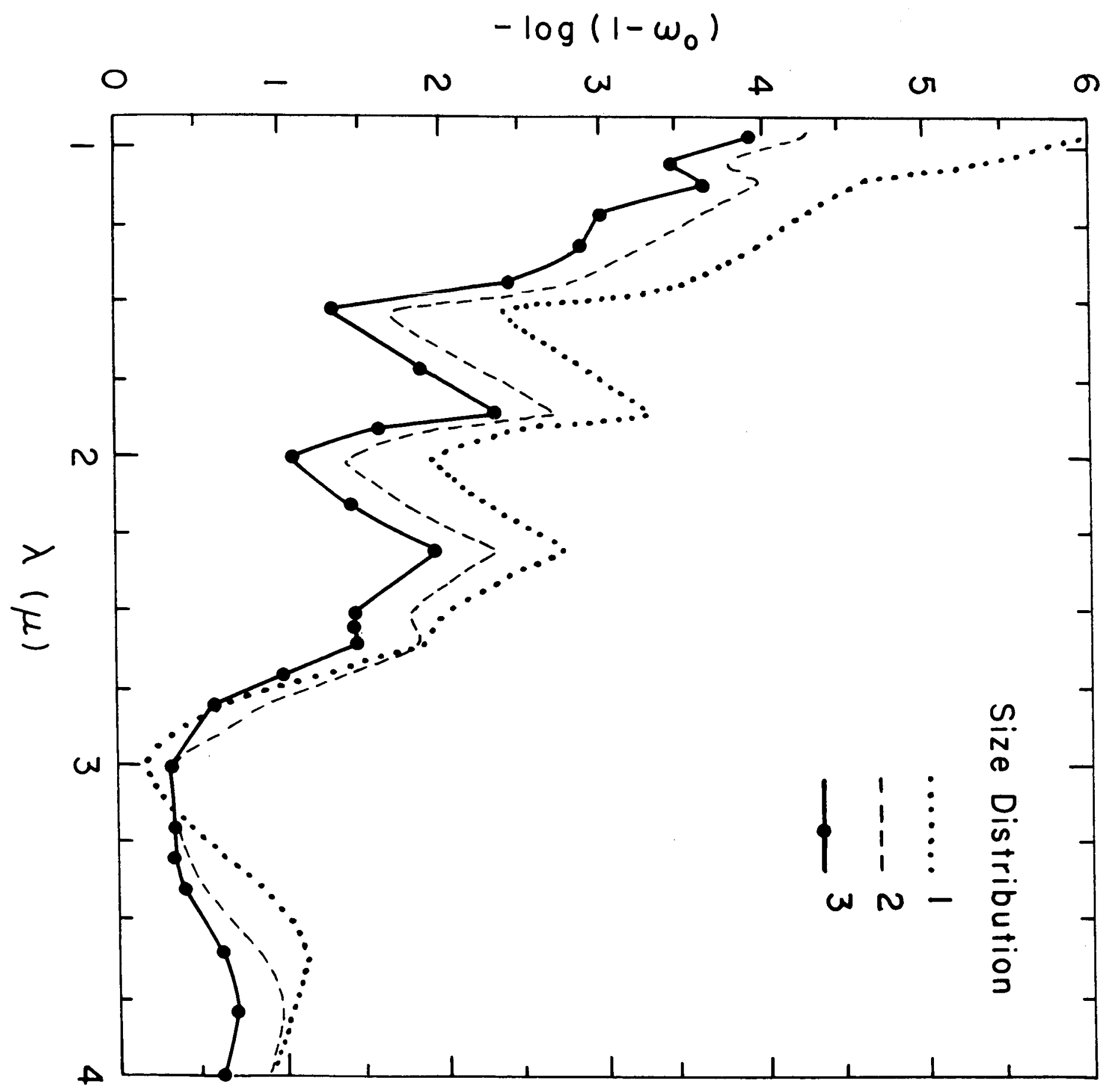

Fig. 2 


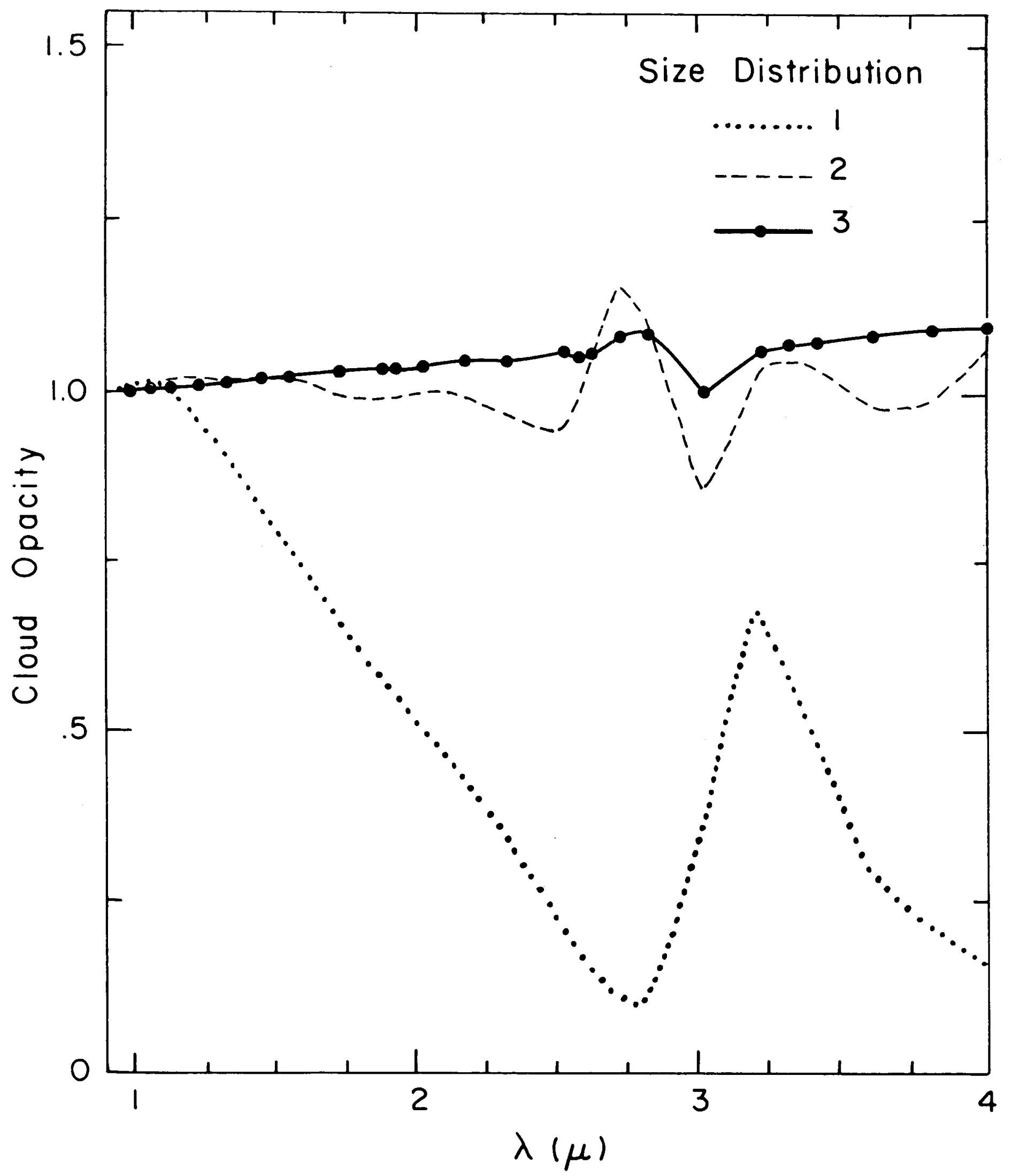

Fig. 3 


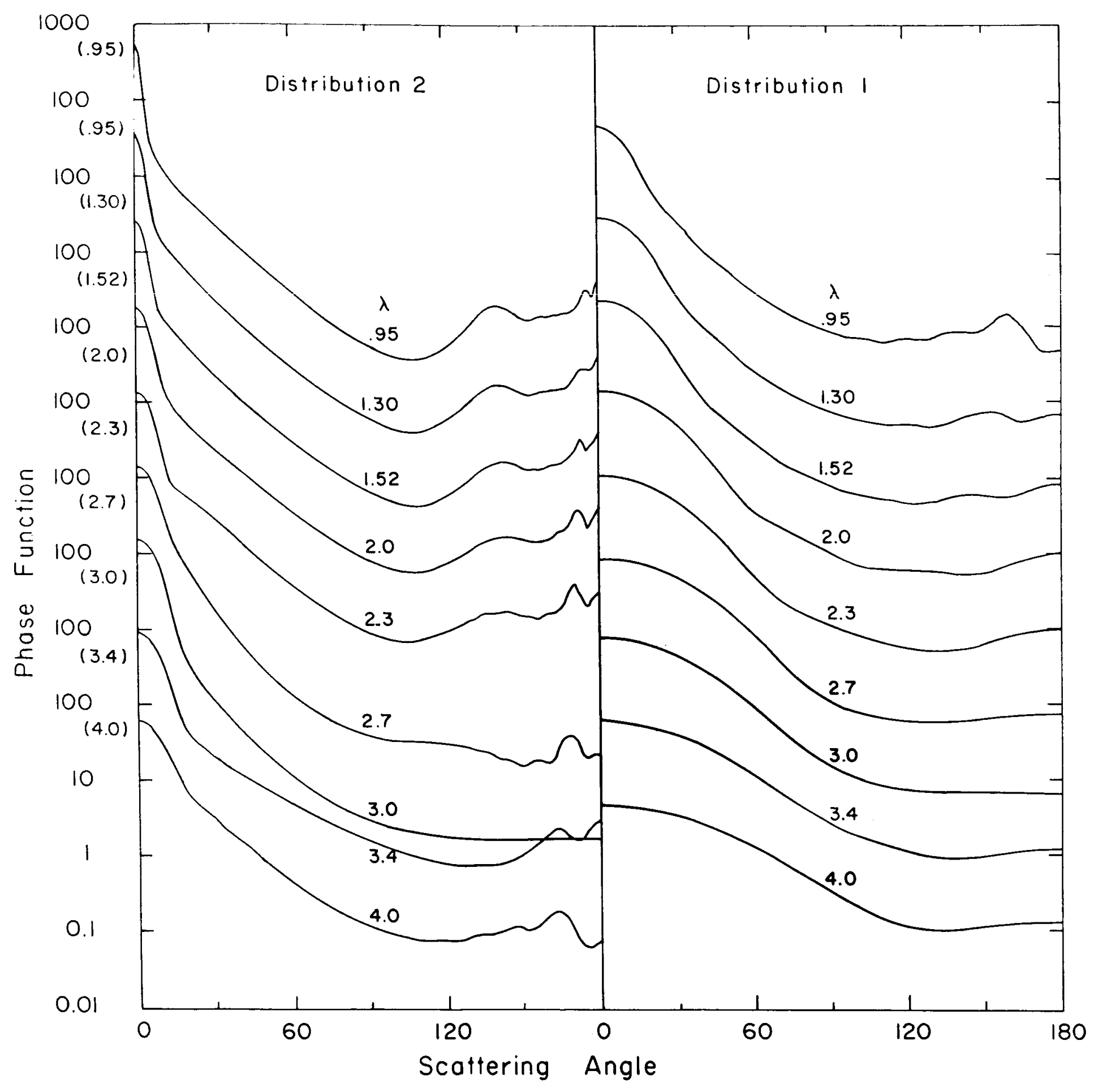

Fig. 4 


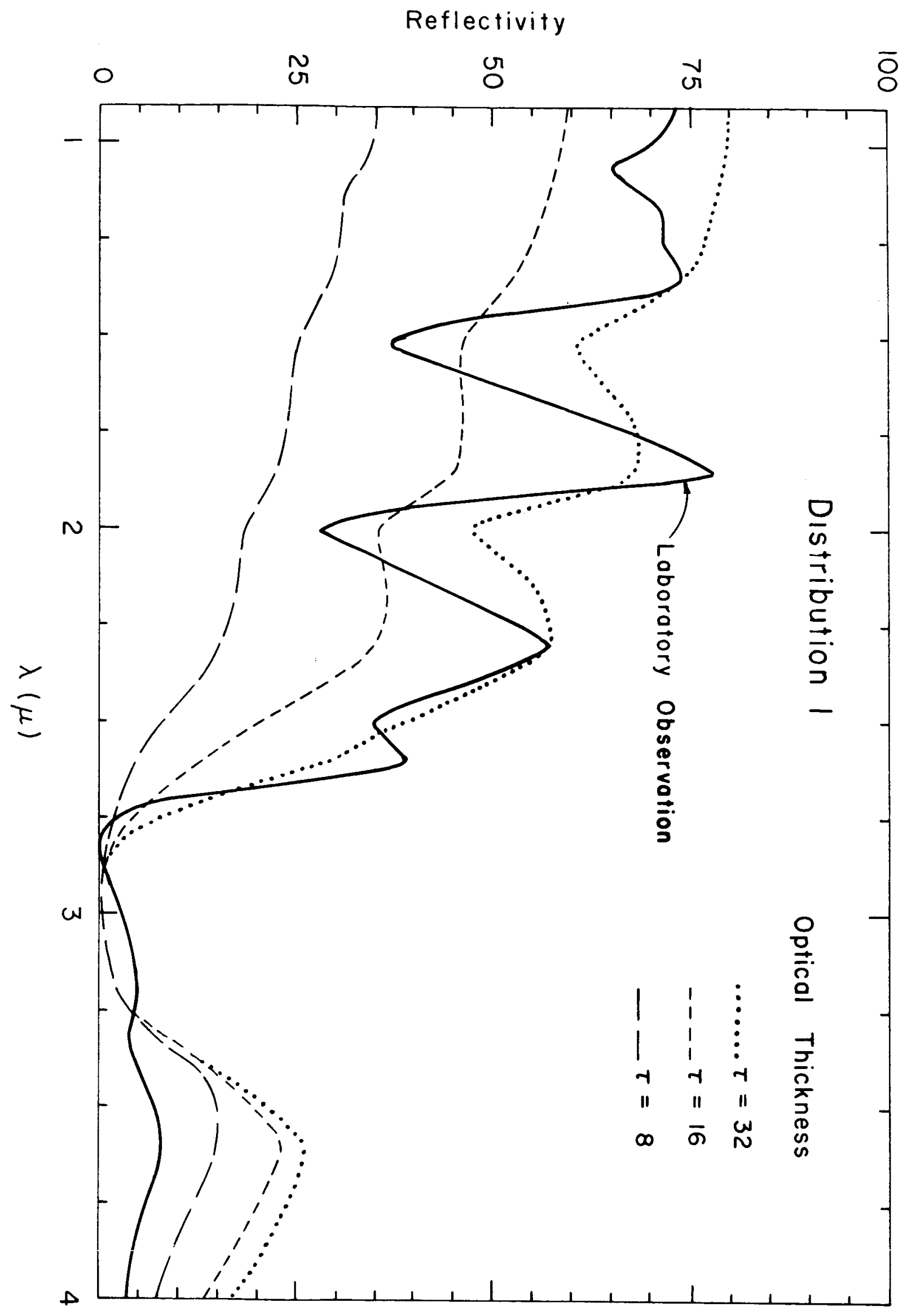

Fig. 5 


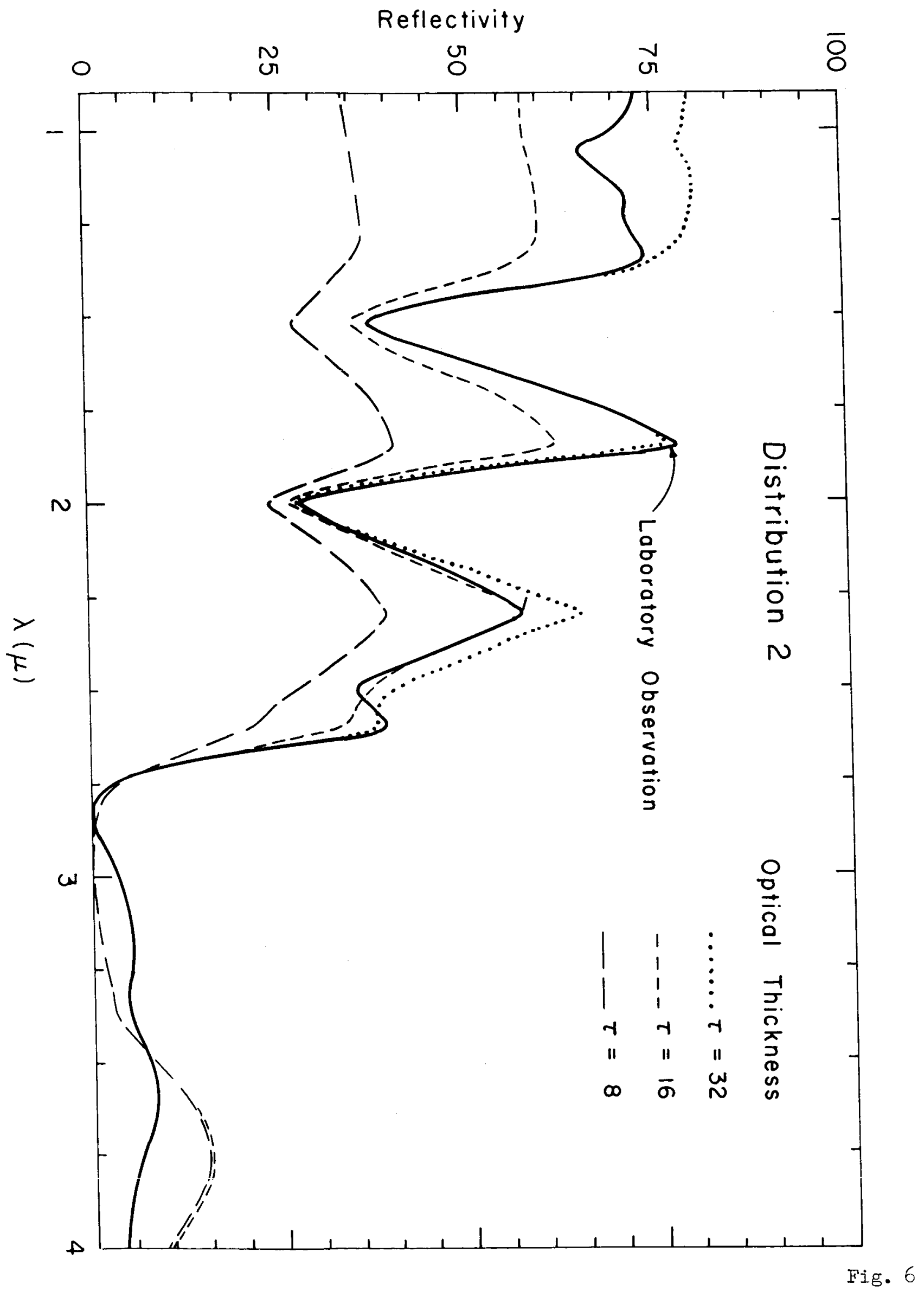






Fig. 7 
Reflectivity

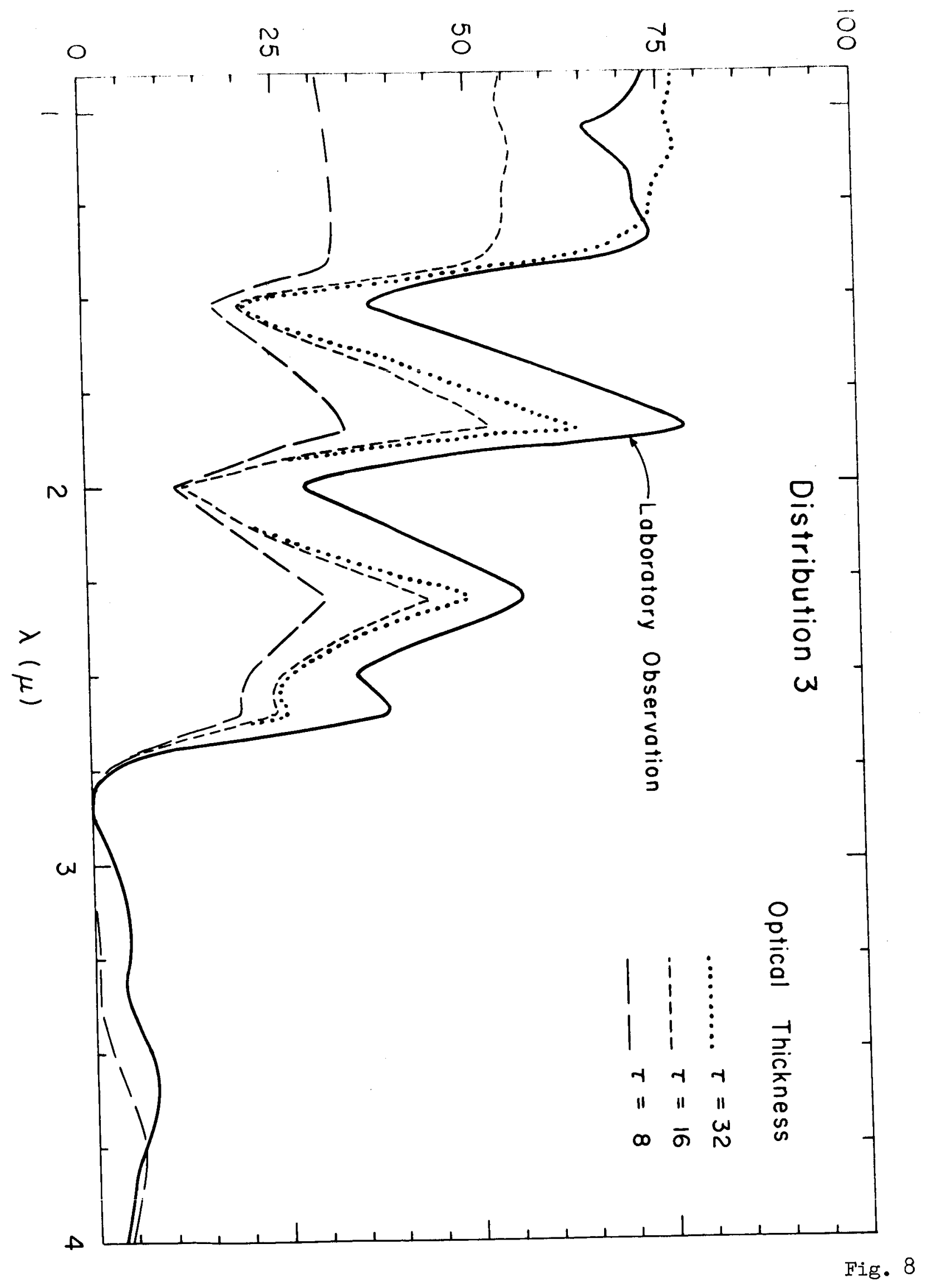

